\title{
Genome-Wide Association Mapping of Resistance to Fusarium Head Blight Spread and Deoxynivalenol Accumulation in Chinese Elite Wheat Germplasm
}

\author{
Lei Wu, Yu Zhang, Yi He, Peng Jiang, Xu Zhang, ${ }^{\dagger}$ and Hongxiang Ma ${ }^{\dagger}$ \\ Institute of Food Crops, Jiangsu Academy of Agricultural Sciences/Jiangsu Collaborative Innovation Center for Modern Crop Production, \\ Nanjing 210014, China \\ Accepted for publication 6 March 2019.
}

\begin{abstract}
Improving resistance to Fusarium head blight (FHB) in wheat is crucial in the integrated management of the disease and prevention of deoxynivalenol (DON) contamination in grains. To identify novel sources of resistance, a genome-wide association study (GWAS) was performed using a panel of 213 accessions of elite wheat germplasm of China. The panel was evaluated for FHB severity in four environments and DON content in grains in two environments. High correlations across environments and high heritability were observed for FHB severity and DON content in grains. The panel was also genotyped with the $90 \mathrm{~K}$ Illumina iSelect single

nucleotide polymorphism (SNP) array and 11,461 SNP markers were obtained. The GWAS revealed a total of six and three loci significantly associated with resistance to fungal spread and DON accumulation in at least two environments, respectively. QFHB-2BL.1 tagged by IWB52433 and QFHB-3A tagged by IWB50548 were responsible for resistance to both fungal spread and DON accumulation. In summary, this study provided an overview of FHB resistance resources in elite Chinese wheat germplasm and identified novel resistance loci that could be used for wheat improvement.
\end{abstract}

Fusarium head blight (FHB) caused by Fusarium graminearum, F. culmorum, F. asiaticum, and other Fusarium spp. is one of the most destructive diseases of wheat worldwide (Bai and Shaner 2004; McMullen et al. 2012). Historically, serious FHB epidemics occurred mainly in the middle to low reaches of the Yangtze River and the northeast spring wheat growing area in China. However, FHB epidemics have extended to the north, southwest, and northwest winter wheat growing areas, especially the Yellow and Huai River valleys, owing to changes in climate and cropping systems (Ma et al. 2008). FHB results in grain yield loss, decreased grain quality, and contamination with mycotoxins. As a predominant mycotoxin associated with FHB infections, deoxynivalenol (DON) causes feed refusal or poor weight gain in animals and may cause immunological or alimentary disorders in humans (Escrivá et al. 2015; McMullen et al. 2012). Guidelines and restrictions for DON levels in cereals or cereal products have been established in most countries, including the United States, European Union, Canada, and China, to control the food security risks (Buerstmayr et al. 2009).

Breeding for resistant varieties is crucial in the integrated management of FHB and prevention of DON contamination in grains. Host resistance to FHB in wheat is a complex quantitative

†Corresponding authors: H. Ma; hongxiangma@163.com, and X. Zhang; xuzhang@jaas.ac.cn

Funding: This study was supported by grants from the National Natural Science Foundation of China (31561143004), National Key R\&D Program of China (2016YFD0101802 and 2016YFE0112900), Jiangsu Provincial Key Technology R\&D Programs, China (BE2015352), and Natural Science Foundation of Jiangsu Province, China (BK20170609).

L. Wu and Y. Zhang contributed equally to this work.

*The $\boldsymbol{e}$-Xtra logo stands for "electronic extra" and indicates that two supplementary figures and three supplementary material files are published online.

The author(s) declare no conflict of interest.

(C) 2019 The American Phytopathological Society trait largely influenced by genotype-by-environment interactions (Fuentes et al. 2005; Ma et al. 2006). Five different types of FHB resistance have been described: type I, resistance to initial infection; type II, resistance to fungal spread; type III, resistance to DON accumulation; type IV, resistance to kernel infection; and type V, tolerance to the disease (Mesterházy 1995; Mesterházy et al. 1999). Most quantitative trait loci (QTLs) for FHB resistance were mapped by using biparental mapping populations with the genetic variants detected between two parental genotypes (Buerstmayr et al. 2002, 2003; Jiang et al. 2007a, b). More than 100 QTLs for different types of FHB resistance have been found on all wheat chromosomes on various resistant sources, but a large number of resistance QTLs are not well characterized and few diagnostic markers for QTLs have been discovered and/or developed, which limits their utility in breeding programs. Fhb1 (type II resistance) was identified in the highly resistant Chinese landraces Sumai3, Wangshuibai, and their derivatives (Liu and Anderson 2003; Miedaner and Korzun 2012; Wilde et al. 2007). It is widely accepted that Fhb1 provides both type II and type III resistance (Buerstmayr et al. 2009). Fhb1 has been introgressed into various breeding populations using phenotypic and marker-assisted selection (MAS) (Anderson et al. 2007; Buerstmayr et al. 2009; del Blanco et al. 2003; Liu et al. 2009). Unique candidate genes for Fhb1 were reported in CM-82036 (Schweiger et al. 2016) and a pore-forming toxin-like gene encoding a chimeric lectin with two agglutinins at the Fhb1 locus was identified to be involved in conferring FHB resistance (Rawat et al. 2016). To date, no other genes for FHB resistance have been cloned and functionally characterized. Therefore, it is essential to identify and characterize more effective and stable resistance genes from different genetic backgrounds for MAS.

Genome-wide association study (GWAS) mapping provides a powerful tool to identify novel loci responsible for complex quantitative traits in germplasm collection using the high-density single nucleotide polymorphism (SNP) array. GWASs have been used to map QTLs for yield traits (Chen et al. 2016; Dodig et al. 2012), milling quality (Breseghello and Sorrells 2006; Reif et al. 2011), preharvest sprouting (Kulwal et al. 2012; Mohan et al. 2009), and disease resistance (Adhikari et al. 2011; Arruda et al. 2016; 
Kollers et al. 2013; Letta et al. 2013; Maccaferri et al. 2015; Miedaner et al. 2011; Naruoka et al. 2015; Yang et al. 2005; Zegeye et al. 2014) in wheat. Miedaner et al. (2011) conducted genomewide association analysis using 455 European soft winter wheat accessions and revealed nine significant loci for FHB resistance on seven chromosomes using 115 simple sequence repeat (SSR) markers. Kollers et al. (2013) reported that consistent associations for type I resistance to FHB were on all chromosomes except $6 \mathrm{~B}$ in 358 European winter wheat accessions. More positive and stable loci were detected when high-density markers such as the 90k SNP assay or genotyping-by-sequencing (GBS) markers were available. Arruda et al. (2016) performed a GWAS for FHB resistance using GBS markers in a panel including 273 accessions from midwestern and eastern regions of the United States and found significant SNPtrait associations on chromosomes 3B, 4A, 4D, 6A, 7A, and 7D for type I resistance, chromosome $3 \mathrm{~B}$ for type II resistance, and chromosomes $1 \mathrm{D}$ and $3 \mathrm{~B}$ for type III resistance, respectively (Arruda et al. 2016). Seventeen consistent significant SNP associations with multiple types of resistance were found on chromosomes $1 \mathrm{~B}, 2 \mathrm{~B}, 4 \mathrm{~B}, 5 \mathrm{~A}, 5 \mathrm{~B}$, and $6 \mathrm{~A}$ in spring wheat lines developed in the Pacific Northwest and by the International Maize and Wheat Improvement Center (CIMMYT) (Wang et al. 2017).

The objectives of this study were to screen a collection of elite wheat germplasm of China in field evaluation and to perform a genome-wide association analysis to identify QTLs for FHB resistance (type II and type III) that are suitable for MAS and further genetic analysis.

\section{MATERIALS AND METHODS}

Plant materials and FHB evaluation. A panel of 213 accessions of elite Chinese wheat germplasm including five landraces, 74 breeding lines, and 134 commercial cultivars (Supplementary Material File S1) was used in this study. These accessions originated from four ecological wheat growing regions: the northern region (NOR), the Yellow and Huai River valley region (YH), the middle to lower Yangtze valley region (YT), and the southwestern region (SW).

FHB resistance was evaluated in two locations (Nanjing $[\mathrm{NJ}]$ and Luhe [LH]) in 2010 and 2011. Each year, all of the accessions were grown as 1.2-m headrows in November and were evaluated for FHB resistance the next April by using the single floret inoculation method. The experiment was set up as a complete randomized block design with two replications. Sumai3 and Yangmai158 were used as resistant or moderately resistant checks, whereas Xiaoyan 22 was used as a susceptible check. Ten to 15 plants per accession were inoculated by injecting $10 \mu \mathrm{l}$ of a conidial suspension of $F$. asiaticum $(100$ conidia/ $\mu \mathrm{l})$ into a central spikelet at early anthesis. The inoculated spikes were covered with a plastic bag for $72 \mathrm{~h}$ to meet the moisture requirement for fungal infection. After the plastic bag was removed, misting irrigation was applied to create a humid environment for fungal spread. The percentage of scabbed spikelets was calculated as FHB severity (SEV) at day 21 after inoculation. The same materials were also evaluated for FHB resistance in NJ in 2012 and 2017 using the same inoculation method. The grains of all infected spikes were harvested to detect the DON content with a DON ELISA test kit (Huaan Magnech Bio-Tech) using an indirect competitive enzyme-labeled immunoassay.

Phenotypic data analysis. The best linear unbiased predictions (BLUPs) for each accession across different environments for FHB SEV and DON content were estimated using $\mathrm{R}$ package "Ime4" in order to combine analysis of variance across environments in a mixed linear model including genotype, environment, and genotype-by-environment interactions as random factors ( $\mathrm{R}$ Development Core Team 2011). The broad-sense heritability $\left(H^{2}\right)$ of SEV and DON was calculated by a random-effect model. Pearson correlation coefficients among environments were computed by R to test the consistency of FHB responses across different environments. Tukey mean comparison tests were performed for SEV and DON among subpopulations or ecological wheat growing regions.

Genotypic data analysis. Genomic DNA was extracted from leaves of 2-month-old seedlings per accession using the Plant Genomic DNA extraction kit (Karroten). The 213 accessions were genotyped using the Illumina iSelect $90 \mathrm{~K}$ wheat SNP assay at Jiangsu Academy of Agricultural Sciences in Nanjing, China. The data filtering steps were as follows: (i) markers with more than $10 \%$ missing data or accessions with more than $10 \%$ missing data were eliminated and (ii) markers with a minor allele frequency $<10 \%$ were eliminated. A total of 11,479 polymorphic SNPs with known map positions according to a consensus of common wheat (Wang et al. 2014) were used for population structure and linkage disequilibrium (LD) analysis.

Population structure, LD estimation, and GWAS. Population structure was analyzed using the Bayesian model-based clustering method in Structure version 2.3.4. Five iterations for hypothetical subpopulations (K value) from 1 to 10 were performed based on an admixture model with the same settings $(100,000$ Markov chain Monte Carlo replications and 10,000 length of burnin period). Tassel version 5.0 was used to calculate the LD squared allele frequency correlation $\left(r^{2}\right)$ estimates for all pairwise comparisons between all of the mapped SNPs. The pairwise LD $r^{2}$ estimates from all loci were plotted with the corresponding pairwise genetic distances, and then a nonlinear regression was fitted in R. The method described by Breseghello and Sorrells (2006) was used to estimate the linked genetic distance as follows: (i) the square root for $r^{2}$ was transformed to approximate a normally distributed random variable and (ii) the parametric 95th percentile of that distribution was taken as a critical value of $r^{2}$. The intersection of the curve fit to $r^{2}$ with this baseline was considered as the estimate of the extent of LD in the chromosome. All calculations were performed in $\mathrm{R}$.

A GWAS of loci responsible for FHB resistance (type II and type III) in all wheat accessions was performed using the compressed mixed linear model (cMLM) implemented in the $\mathrm{R}$ package Genome Association and Prediction Integrated Tool (GAPIT) (Lipka et al. 2012; Zhang et al. 2010b). The kinship matrix (K matrix) and Q matrix were calculated by Tassel version 5.0 and Structure version 2.3.4, respectively. The K matrix was considered as a random-effect factor and the Q matrix was a covariate to correct for the population structure in association analysis. Model cMLM was used in the analysis because of its improved statistical power (Zhang et al. 2010b). Data sets from the single environment and BLUP values across the different environments were used for association analysis. Markers that were significant at $P<0.001$ in at least two environments were considered to be a stable locus. The physical positions of candidate SNP or SSR markers were obtained from the International Wheat Genome Sequencing Consortium website (http://www.wheatgenome.org) to align the associated SNPs to known FHB resistant genes/QTLs.

\section{RESULTS}

Phenotype analysis across environments. Distributions of SEV and DON were skewed toward resistance in all environments except LH2010 (Fig. 1A to F). Substantial phenotypic variation for both traits was observed in all environments. Mean SEV ranged from 37.35 to $60.86 \%$, and mean DON ranged from 13.23 to 26.78 $\mu \mathrm{g} / \mathrm{kg}$. According to the BLUPs for SEV across four environments (Fig. 1G), 50 accessions (23.26\%), including Wangshuibai, Sumai3, Ning894037, Ningmai6, Shengxuan6, and Ningmai20, were highly resistant (mean SEV $=18.56 \%$ ), whereas 62 accessions $(28.84 \%)$ were highly susceptible to FHB (mean SEV $=62.02 \%)$. In addition, DON content in several accessions, including Ning894037, Wannian2, Wangshuibai, Sumai3, W32, Ning7840, Ningmai8, and Hong5002, was $<3 \mu \mathrm{g} / \mathrm{kg}$ based on the BLUP value for DON content. Broad sense heritability $\left(\mathrm{H}^{2}\right)$ values for SEV and 
DON content were estimated as 0.93 and 0.56 across the different environments. SEV was significantly correlated with DON content in different environments at $P=0.001$, ranging from 0.41 to 0.89 (Table 1; Supplementary Fig. S1).

Population structure and kinship. The hierarchical ward clustering method implemented in GAPIT indicated that the population structure of the panel had two major groups, which could be further divided into six subgroups (1A, 1B, 1C, 2A, 2B, and 2C) (Fig. 2A and B). The subgroup membership coefficients analysis showed a moderate degree of admixture between cultivars and breeding lines (Fig. 2C). A high proportion of accessions from $\mathrm{YH}(44 / 53)$ were clustered into group 1 and $66 \%$ of accessions from YT $(95 / 143)$ were clustered into group 2. Subgroup 1A included eight accessions from YT, mainly of which were Ningmai series
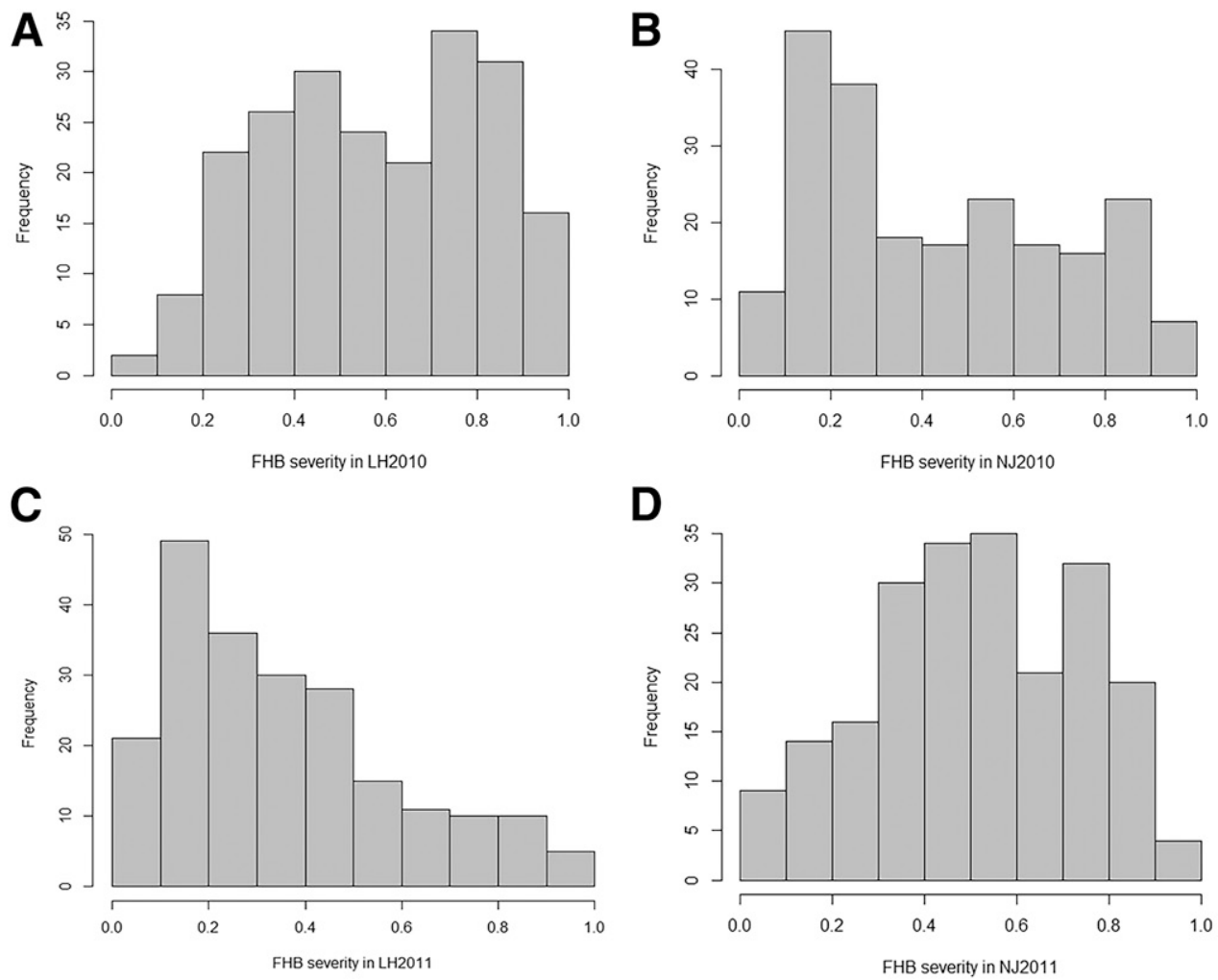

D
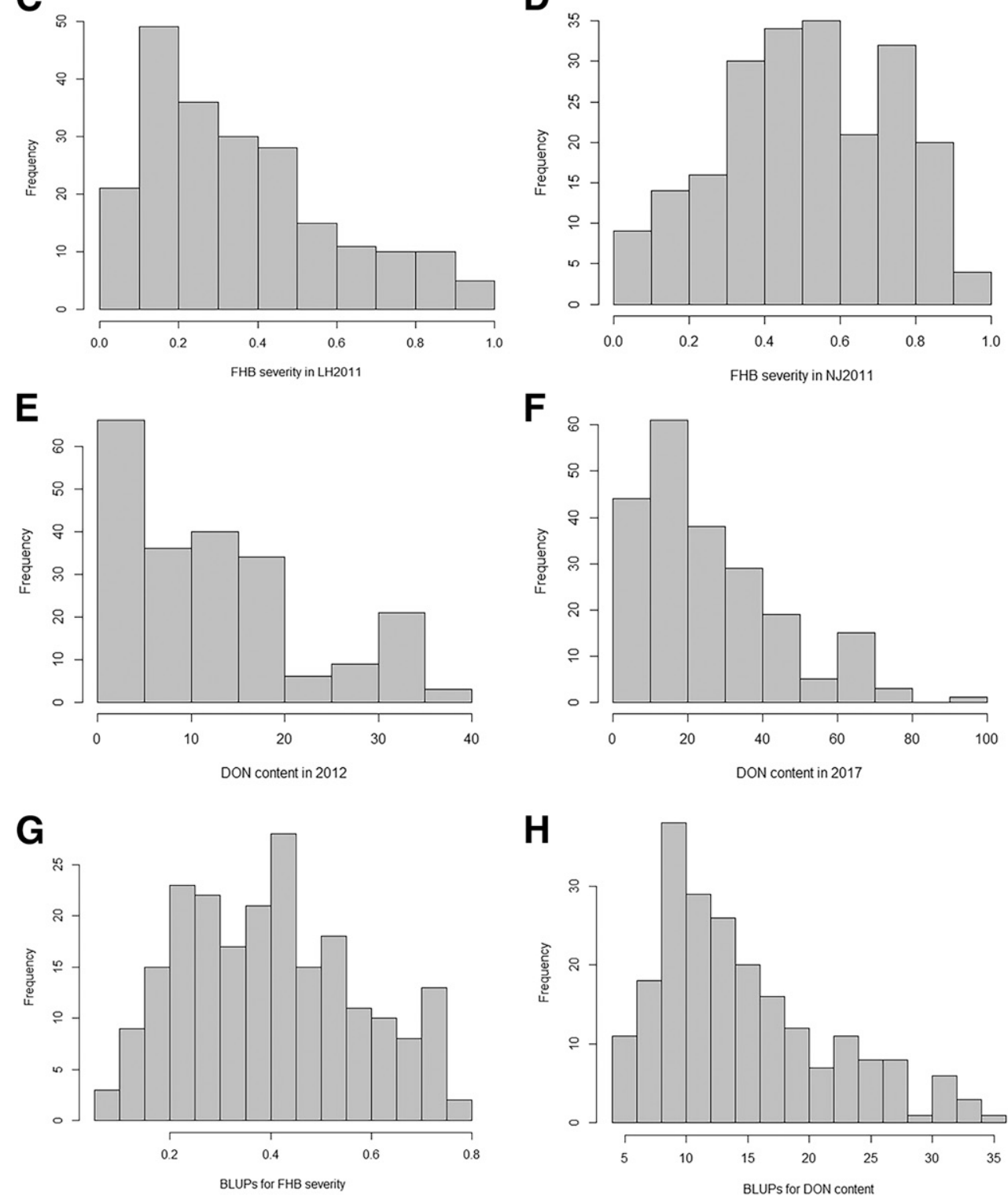

Fig. 1. Distributions of A, B, C, and D, Fusarium head blight (FHB) severity and $\mathbf{E}$ and F, deoxynivalenol (DON) content of 213 wheat accessions in response to Fusarium asiaticum and $\mathbf{G}$ and $\mathbf{H}$, best linear unbiased predictions (BLUPs) across different environments. LH2010, NJ2010, LH2011, and NJ2011 represent the different environments: Luhe in 2010, Nanjing in 2010, Luhe in 2011, and Nanjing in 2011, respectively. 
released by Jiangsu Academy of Agricultural Sciences. All of the accessions in subgroup 2A were from Jiangsu province except Mian2000-1-37 (Fig. 2D; Supplementary Material File S1).

In addition, accessions in different subgroups or wheat growing regions displayed significantly different resistance levels to fungal spread and DON contamination (Fig. 3). The mean level of FHB resistance was higher in subgroup $2 \mathrm{~A}$ than that in subgroups $1 \mathrm{~B}$ and 1C $(P<0.05)$ (Fig. 3A and B). In subgroup 2A, $47.37 \%$ of the accessions were moderately resistant to $\mathrm{FHB}$, whereas only 22.41 and $11.90 \%$ of the accessions in subgroups $1 \mathrm{~B}$ and $1 \mathrm{C}$ were moderately resistant to $\mathrm{FHB}$, respectively. In addition, the mean level of FHB resistance was higher in YT than that in the other groups $(P<0.05)$ (Fig. 3C and D). The results showed that $30.07 \%$ of the accessions from YT were moderately resistant to FHB, but only $9.43 \%$ of the accessions from $\mathrm{YH}$ had moderate FHB resistance.

Genome-wide LD estimation. The LD analysis was based on pairwise squared correlations $\left(r^{2}\right)$ for all 11,479 mapped SNP markers. Genome-wide LD generally declined with the genetic distance by fitting the smoothing spline curve of the scatter plot of the $r^{2}$ distribution (Fig. 4). The horizontal dashed lines represent the baseline $r^{2}$ value $(0.13)$ based on the 95 th percentile of the distribution of $r^{2}$ values between pairs of unlinked markers. The confidence intervals for QTLs were $4 \mathrm{cM}$ corresponding to the critical $r^{2}$ of 0.13 . The median $r^{2}$ value for pairs of completely linked markers was 0.35 , with interquartile values ranging from 0.05 to 0.88 (Fig. 4B). The $\mathrm{LD}$ values for paired markers decayed to 0.13 at the genetic distance ranging from 0.1 to $1.0 \mathrm{cM}$ and dramatically decreased to 0.05 within 1.1 to $5.0 \mathrm{cM}$. The $\mathrm{LD}$ value decreased slowly when genetic distance was more than $5 \mathrm{cM}$, and no significant differences were observed after the genetic distance reached $20 \mathrm{cM}$.

Association analysis for FHB resistance across environments. Maker-trait association (MTA) was carried out for each environment using all 213 accessions in the elite Chinese wheat panel to identify the loci responsible for FHB resistance. In total, 21 and 17 loci on 10 chromosomes were significantly associated $(P<0.001)$ with type II and III resistance, respectively (Supplementary Fig. S2). Seven loci, including QFHB-1A, QFHB2BL.1, QFHB-2BL.2, QFHB-3A, QFHB-3B.1, QFHB-3B.2, and QFHB-7A, on chromosomes 1A, 2B, 3A, 3B, and 7A showed a significant association with type II or III resistance in at least two environments (Table 2; Supplementary Material File S2). The cumulative phenotypic variation of type II and III resistance explained by multiple loci was 43.5 and $16.3 \%$, respectively.

TABLE 1. Pearson correlation coefficients of Fusarium head blight (FHB) severity and deoxynivalenol (DON) content of 213 wheat accessions across different environments $^{\mathrm{z}}$

\begin{tabular}{|c|c|c|c|c|c|c|c|}
\hline & SEVLH2010 & SEVNJ2010 & SEVLH2011 & SEVNJ2011 & BLUPSEV & DON2012 & DON2017 \\
\hline \multicolumn{8}{|l|}{$\overline{\text { SEVLH2010 }}$} \\
\hline SEVNJ2010 & 0.74 & & & & & & \\
\hline SEVLH2011 & 0.60 & 0.64 & & & & & \\
\hline BLUPSEV & 0.87 & 0.89 & 0.84 & 0.84 & & & \\
\hline DON2012 & 0.53 & 0.62 & 0.60 & 0.71 & 0.72 & & \\
\hline DON2017 & 0.42 & 0.41 & 0.56 & 0.45 & 0.53 & 0.46 & \\
\hline BLUPDON & 0.53 & 0.56 & 0.66 & 0.63 & 0.69 & 0.75 & 0.93 \\
\hline
\end{tabular}

z SEVLH2010, SEVNJ2010, SEVLH2011, and SEVNJ2011 represent mean FHB severities evaluated in the Luhe nursery in 2010, Nanjing in 2010, Luhe in 2011, and Nanjing in 2011, respectively. BLUPSEV and BLUPDON represent the best linear unbiased predictions (BLUPs) for FHB severity and DON content across different environments. DON2012 and DON2017 represent mean DON contents detected from grains harvested in the Nanjing nursery in 2012 and 2017 , respectively. $P$ values of the Pearson correlation coefficients shown in the table are $<0.001$.

\section{A Clusteringstructure}

B Genetic similarity

C Q matrix
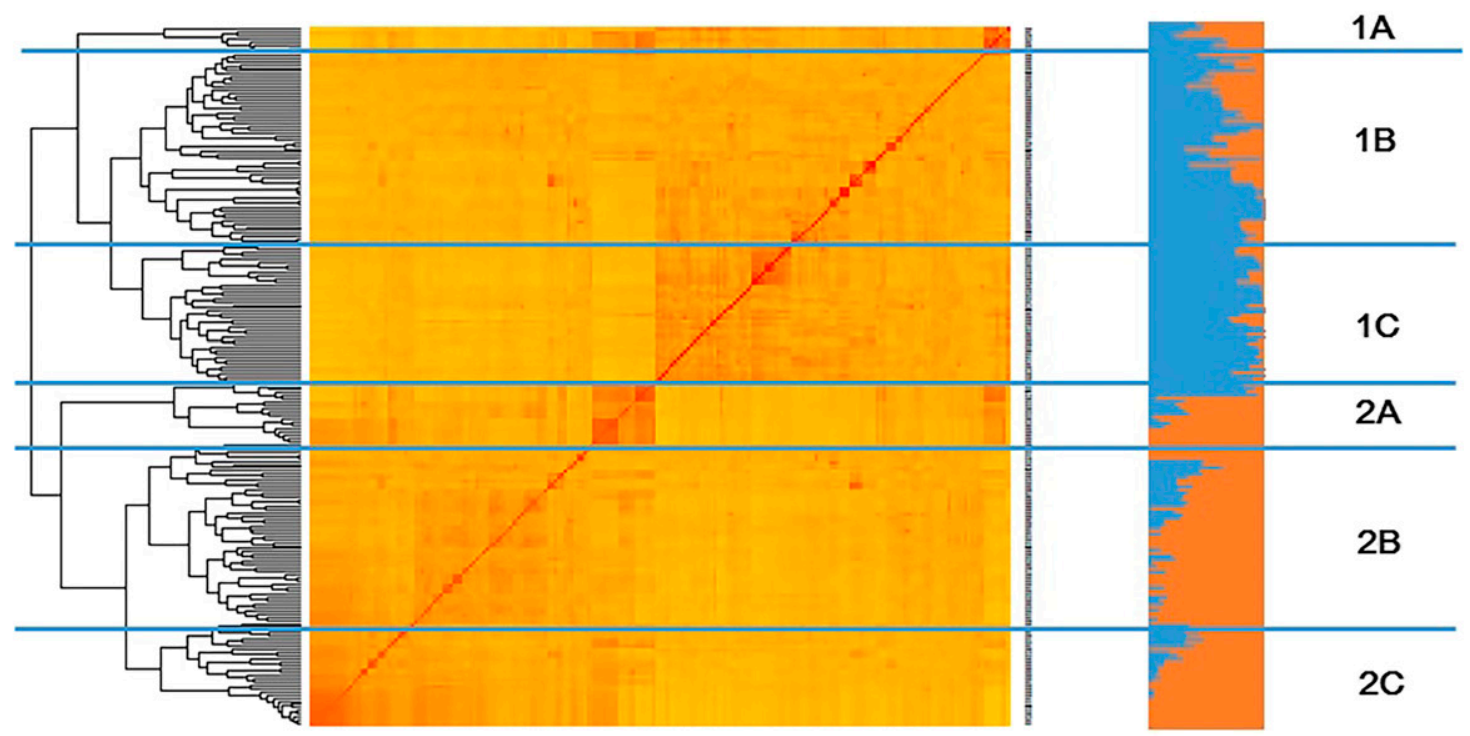

D Origin

Fig. 2. Population structure and kinship analysis of 213 elite wheat accessions. A, Clustering analysis of 213 accessions based on the genotype data. Horizontal lines are used to classify accessions into two main groups and six subgroups. B, Kinship matrix for all 213 accessions. C, Membership coefficients of the accessions derived from the Structure analysis $(\mathrm{Q}=2)$. D, Percentage of origins of the accessions in subgroups: northern region (NOR), Yellow and Huai River valley region $(\mathrm{YH})$, middle to lower Yangtze valley region $(\mathrm{YT})$, and southwestern region $(\mathrm{SW})$. 
A

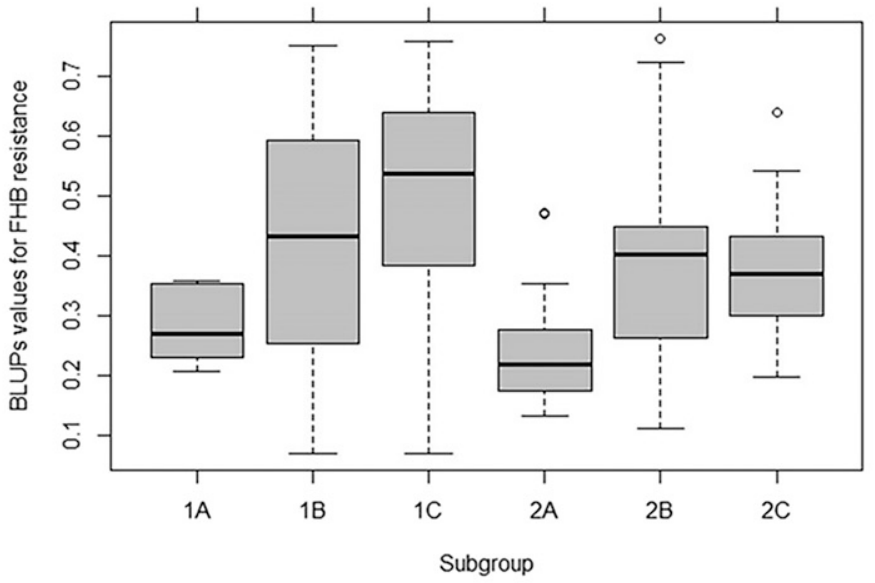

C

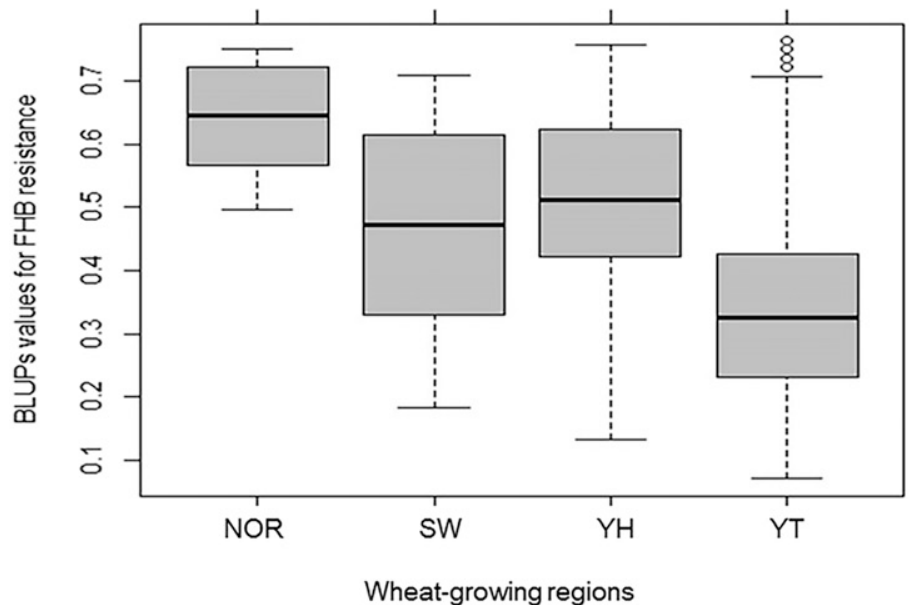

$\begin{array}{lllllll}\mathbf{B} & \begin{array}{lllll}a \\ b\end{array} & b & c & a & \begin{array}{l}a \\ b\end{array} & \begin{array}{c}a \\ b\end{array}\end{array}$

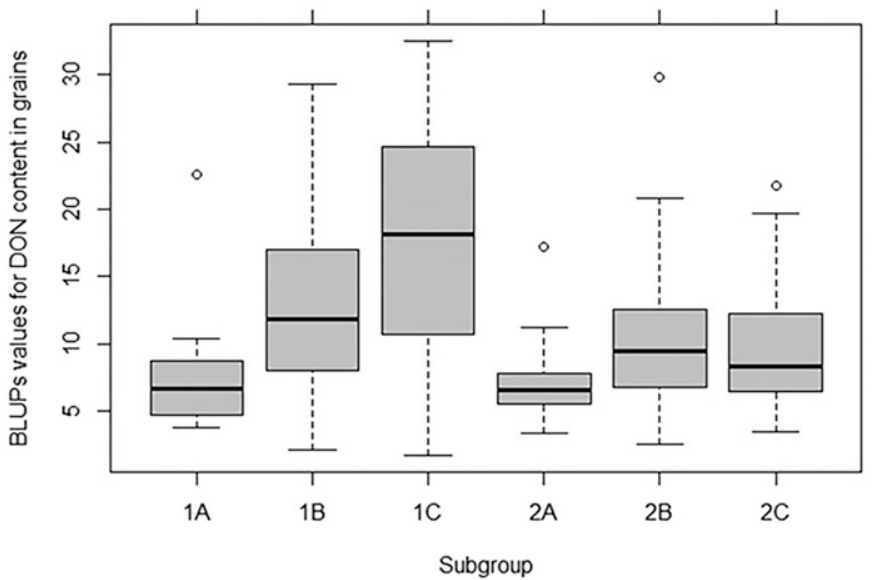

a

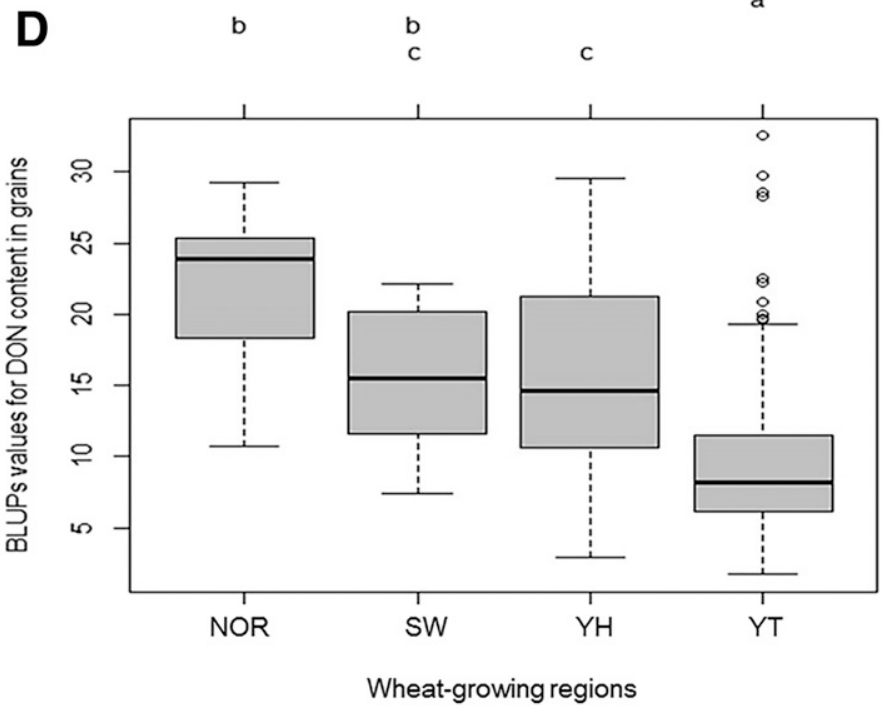

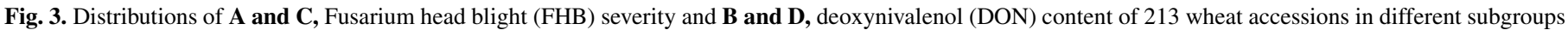

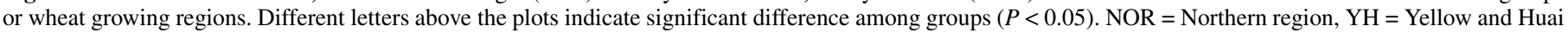
River valley region, YT $=$ middle to lower Yangtze valley region, $\mathrm{SW}=$ southwestern region, and BLUP $=$ best linear unbiased prediction .
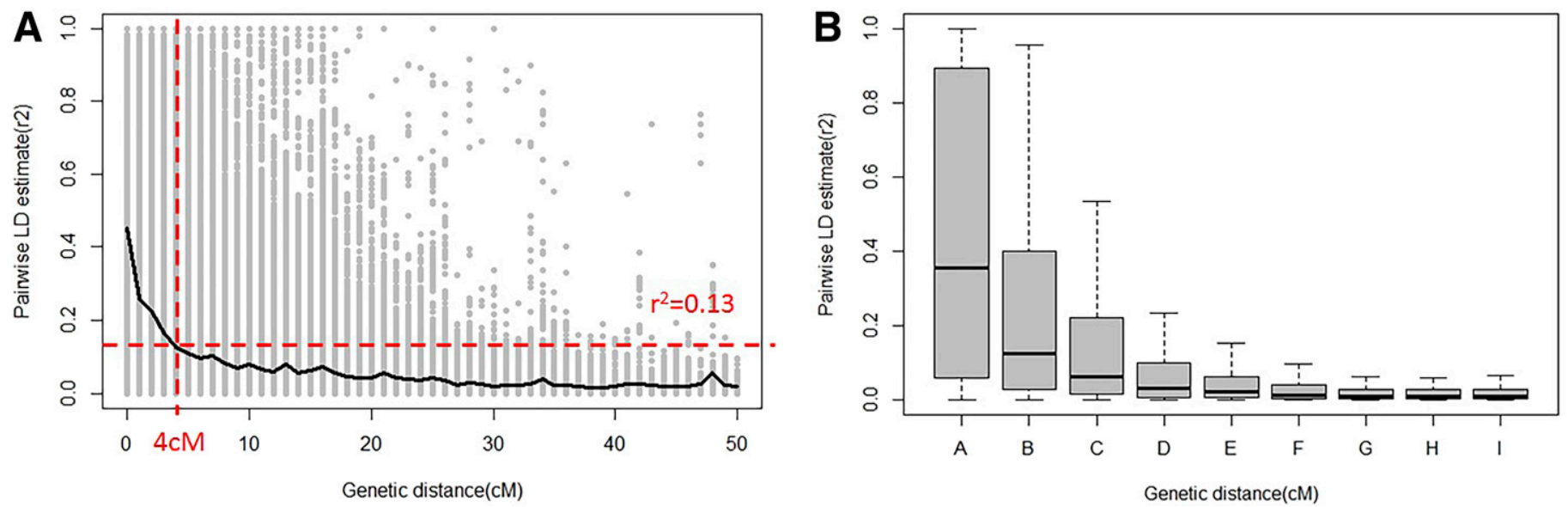

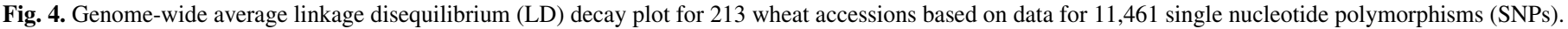

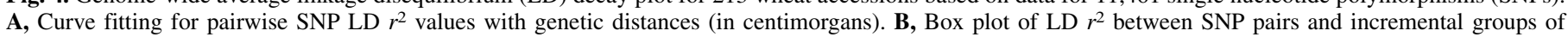

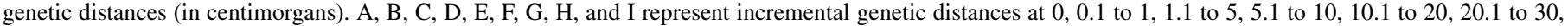
30.1 to $40,40.1$ to 50 , and $>50 \mathrm{cM}$. 
QFHB-2BL, an FHB resistance cluster including 24 MTAs, was identified on the long arm of chromosome $2 \mathrm{~B}$ and could be split into two resistance loci (QFHB-2BL.1 and QFHB-2BL.2) based on LD analysis. QFHB-1A (covering markers IWB24089, IWB8898, IWB51523, IWA3867, IWA4995, and IWB20954), QFHB-3B.1 tagged by IWB12053, and QFHB-3B.2 tagged by IWB10842 were associated with type II resistance. QFHB-7A (covering markers IWA3423, IWB9120, IWB72124, IWB43131, IWB38566, IWB29268, IWA6608, IWA6588, IWA3928, IWA3423, and IWA1339) was associated with type III resistance and explained phenotypic variation ranging from 3.6 to $5.2 \%$. QFHB-2BL.1 tagged by IWB52433 and QFHB3A tagged by IWB50548 were responsible for both type II and III resistance.

The frequency distribution of favorable alleles of FHB resistance in six subgroups and four agroecological wheat growing regions is shown in Table 3. Subgroups $1 \mathrm{~A}, 2 \mathrm{~A}, 2 \mathrm{~B}$, and $2 \mathrm{C}$ had higher frequencies of associated favorable alleles than $1 \mathrm{~B}$ and $1 \mathrm{C}$ for QFHB-1A, QFHB-2BL.1, QFHB-2BL.2, and QFHB-7A. The favorable alleles of QFHB-2BL.2 and QFHB-7A were almost fixed in all of the subgroups. The frequency of favorable alleles of QFHB$1 \mathrm{~A}$ was higher than other alleles in subgroups $2 \mathrm{~B}$ and $2 \mathrm{C}$. The favorable alleles of QFHB-1A, QFHB-2BL.1, and QFHB-2BL.2 were predominant alleles in regions $\mathrm{YH}$ and $\mathrm{YT}$.

Relationship between the number of favorable alleles and FHB resistance. The effects of the number of favorable alleles on BLUP values for FHB SEV and DON content in all 213 wheat accessions were investigated (Fig. 5). With the number of the resistant loci increased, BLUP values of FHB SEV and DON content were significantly decreased $(P<0.001)$, and the regression coefficients were 0.42 and 0.41 , respectively. The results suggested that pyramiding the favorable alleles derived from the association analysis could be useful for improving FHB resistance in wheat cultivars to control FHB epidemics and reduce DON contamination in grains.

\section{DISCUSSION}

Numerous studies of genetic mapping for FHB resistance were reported during the past 2 decades to identify the resistant QTLs or genes in common wheat. However, wheat varieties from the NOR, $\mathrm{YH}$, and SW regions in China with potentially valuable loci have not been well investigated thus far. In this study, wheat germplasm collected from the NOR, YH, YT, and SW regions in China was screened to identify new resistant sources. Field screening of all of the accessions across different environments revealed that FHB SEV ranged from 7.1 to $76.3 \%$, and the resistance levels (type II and III) of accessions from YT were generally higher than those from NW, NOR, and YH $(P<0.05)$ (Fig. 3C and D). In the elite wheat panel, five cultivars (Qu4, Shandong9625, Wan9926, Shannong12, and Zhengmai9023) from the $\mathrm{YH}$ region showed high resistance to FHB.

In four environments in Nanjing, we evaluated FHB resistance using the single floret inoculation method in the field (Fig. 1; Table 1) to simplify the complicated disease system (Buerstmayr

TABLE 2. Quantitative trail loci (QTLs) significantly $(P<0.001)$ associated with Fusarium head blight (FHB) resistance to fungal spread and deoxynivalenol (DON) accumulation in at least two environments (best linear unbiased prediction [BLUP] values included)

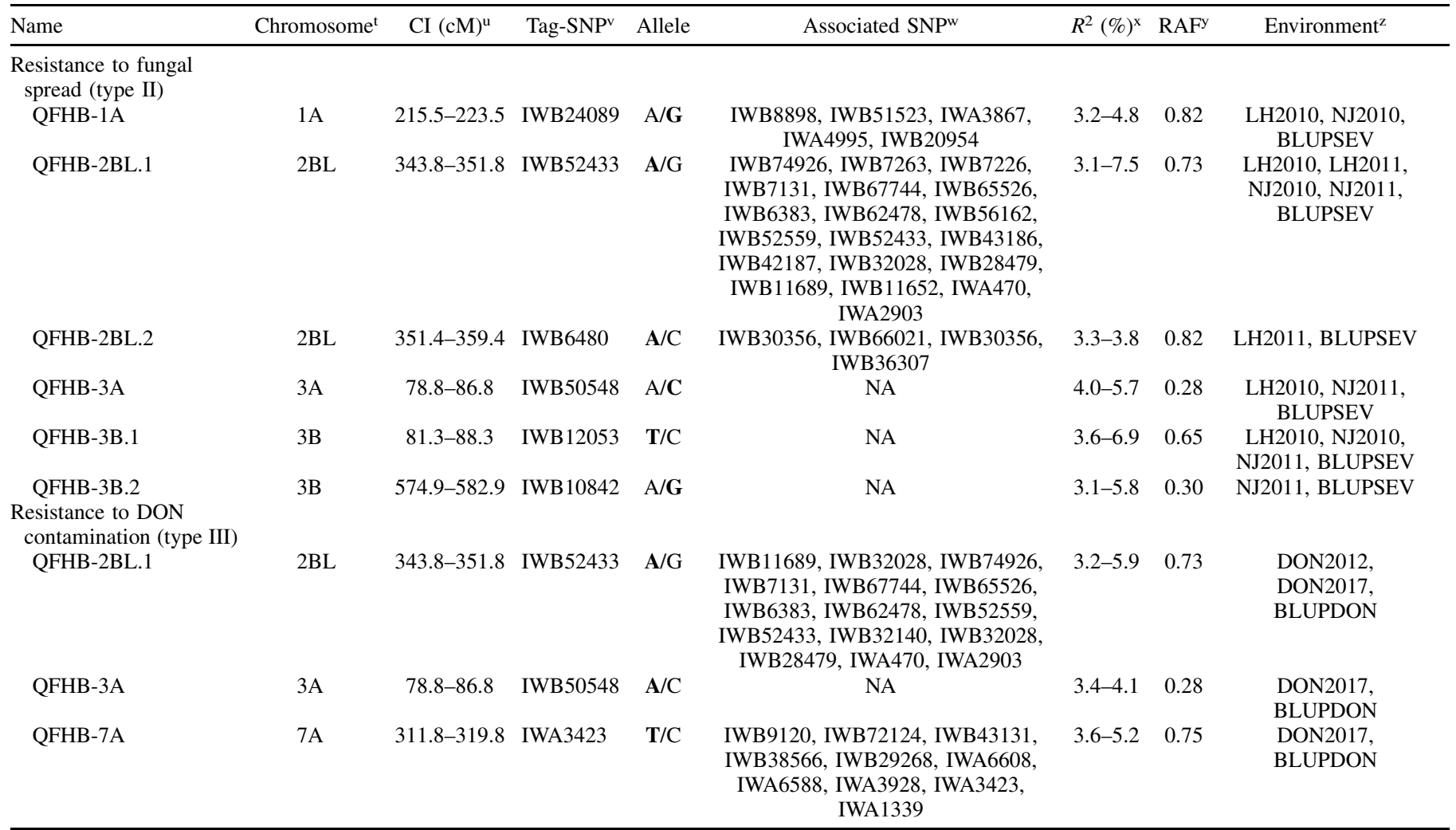

\footnotetext{
${ }^{\mathrm{t}}$ Chromosomes for associated QTLs based on the wheat consensus map for the Illumina iSelect 90K wheat assay (Wang et al. 2014).

" Confidence interval (CI) for associated QTLs derived from the $\pm 4 \mathrm{cM}$ genetic distance of the tagged single nucleotide polymorphism (SNP).

${ }^{v}$ Tag-SNP refers to the significant SNPs with the smallest marker-trait association $P$ values. Resistance alleles of tagged SNPs are shown in bold.

${ }^{\mathrm{w}} \mathrm{NA}=$ not available.

${ }^{x} R^{2}$ explains the phenotypic variances across the environments by tagged SNPs.

y RAF refers to the resistance allele frequencies of the tagged SNPs.

${ }^{z}$ SEVLH2010, SEVNJ2010, SEVLH2011, and SEVNJ2011 represent mean FHB severity evaluated in the Luhe nursery in 2010, Nanjing nursery in 2010, Luhe nursery in 2011, and Nanjing nursery in 2011, respectively. DON2012 and DON2017 represent mean DON content detected in grains harvested from the Nanjing nursery in 2012 and 2017, respectively. BLUPSEV and BLUPDON represent the BLUPs for FHB severity and DON content across different environments, respectively.
} 
et al. 2002). High heritability values for SEV and DON content across environments in our tests indicated the reliability of this FHB evaluation method. This was in agreement with previous reports on mapping of type II resistance (Bai and Shaner 2004; Buerstmayr et al. 2002, 2003, 2009; Jiang et al. 2007b). SEV and DON across the environments were correlated $(P<0.001)$, indicating that loci for type II and III resistance might partially overlap. However, the multilocation and multiyear evaluations were essential for identifying FHB resistance loci in wheat accessions. The BLUPs for SEV and DON across different environments were used to combine analysis of variance across environments in a mixed linear model including genotype, environment, and genotype-by-environment interactions as random factors (Fig. 1). BLUP values were used in the association analysis in order to improve the stability of the results, and MTAs were considered to be significant when they were significant in at least two environments or in the multienvironmental BLUP analysis.

The accessions were unequally distributed among six subgroups, which showed a moderate level of admixture of geographic origins. Frequent exchange of genetic materials in different breeding programs might result in the complex population structure in the present analysis (Fig. 2D). Different accessions were typically grown in distinct agroecological wheat growing regions characterizing different climatic patterns for maximum grain production, and genetic variations were selected for localized adaptation. Regional adaptation might be a crucial determinant for subgroup distribution, as reported in previous studies regarding different types of traits
(White et al. 2008; Zhang et al. 2010a). Therefore, the population used in this study was structured according to agroecological origin and genetic architecture and accessions in different subgroups or wheat growing regions displayed significantly different resistance levels to fungal spread and DON contamination (Fig. 3). All of the accessions in subgroup 2A were from the YT region except Mian2000-1-37 (Fig. 2D), and the accessions in subgroup 2A were generally more resistant (types II and III) than those in subgroups $1 \mathrm{~B}$ and $1 \mathrm{C}$.

Seven loci on chromosomes 1A, 2B, 3A, 3B, and 7A showed a significant association with type II or III resistance in at least two environments. According to the genetic map reviewed by Buerstmayr et al. 2009, at least two QTLs for FHB resistance were identified on chromosome 1A. QFhs.nau-1AS derived from Chinese spring wheat CJ9306 and a major QTL derived from Norwegian spring wheat breeding line NK93604 on chromosome 1A explained 9.5 and $27.9 \%$ of the phenotypic variation for type II resistance, respectively (Buerstmayr et al. 2009). Their physical locations were different from QFHB-1A (tagged SNP: IWB24089; physical location: 301.8 to $347.6 \mathrm{Mb}$ ), the QTL we detected on chromosome 1A. Therefore, QFHB-1A might represent a novel QTL for type II resistance on chromosome 1A, explaining phenotypic variation ranging from 3.2 to $4.8 \%$ (Table 2; Supplementary Material 3).

Two resistant loci (QFHB-2BL.1 and QFHB-2BL.2) including 24 MTAs were detected on the long arm of chromosome 2B. QFHB2BL.1 (594.1 to $681.5 \mathrm{Mb}$ ) was associated with type II and III

TABLE 3. Frequencies of favorable alleles for Fusarium head blight (FHB) resistance quantitative trail loci (QTLs) in subgroups and ecological wheat growing regions

\begin{tabular}{|c|c|c|c|c|c|c|c|c|c|c|c|c|c|}
\hline \multirow[b]{2}{*}{ QTL } & \multirow[b]{2}{*}{ Tag-SNPv } & \multirow[b]{2}{*}{ Allelew } & \multirow[b]{2}{*}{$\mathrm{RAF}^{\mathrm{x}}$} & \multicolumn{6}{|c|}{ Subgroup $^{y}$} & \multicolumn{4}{|c|}{ Wheat growing region ${ }^{z}$} \\
\hline & & & & $1 \mathrm{~A}$ & $1 \mathrm{~B}$ & $1 \mathrm{C}$ & $2 \mathrm{~A}$ & $2 \mathrm{~B}$ & $2 \mathrm{C}$ & NOR & $\mathrm{YH}$ & YT & SW \\
\hline QFHB-1A & IWB24089 & $\mathrm{A} / \mathbf{G}$ & 0.82 & 1 & 0.75 & 0.52 & 1 & 0.96 & 0.97 & 0.38 & 0.7 & 0.92 & 0.56 \\
\hline QFHB-2BL.1 & IWB52433 & $\mathbf{A} / \mathrm{G}$ & 0.73 & 1 & 0.59 & 0.33 & 1 & 0.96 & 0.91 & 0 & 0.42 & 0.92 & 0.22 \\
\hline QFHB-2BL.2 & IWB6480 & $\mathrm{A} / \mathrm{C}$ & 0.82 & 1 & 0.76 & 0.74 & 1 & 0.89 & 0.81 & 0.25 & 0.75 & 0.9 & 0.78 \\
\hline QFHB-3A & IWB50548 & $\mathrm{A} / \mathrm{C}$ & 0.28 & 0.63 & 0.37 & 0.17 & 0.84 & 0.2 & 0.16 & 0.38 & 0.17 & 0.35 & 0.22 \\
\hline QFHB-3B.1 & IWB12053 & $\mathbf{T} / \mathrm{C}$ & 0.65 & 0.75 & 0.42 & 0.26 & 0.79 & 0.13 & 0.13 & 0.5 & 0.34 & 0.29 & 0.22 \\
\hline QFHB-3B.2 & IWB10842 & $\mathrm{A} / \mathbf{G}$ & 0.3 & 0.88 & 0.29 & 0.38 & 0.89 & 0.11 & 0.13 & 0.38 & 0.28 & 0.31 & 0.22 \\
\hline QFHB-7A & IWA3423 & $\mathbf{T} / \mathrm{C}$ & 0.75 & 0.88 & 0.76 & 0.57 & 0.53 & 0.89 & 0.88 & 0.5 & 0.6 & 0.83 & 0.78 \\
\hline
\end{tabular}

v Tag-SNP refers to the significant single nucleotide polymorphisms (SNPs) with the smallest marker-trait association $P$ values.

${ }^{w}$ Resistance alleles of tagged SNPs are shown in bold.

$x$ RAF refers to the resistance allele frequencies of the tagged SNPs.

y Chromosomes for associated QTLs based on the wheat consensus map for the Illumina iSelect 90K wheat assay.

$\mathrm{z}$ NOR $=$ northern region, $\mathrm{YH}=$ Yellow and Huai River valley region, YT $=$ middle to lower Yangtze valley region, and SW $=$ southwestern region .

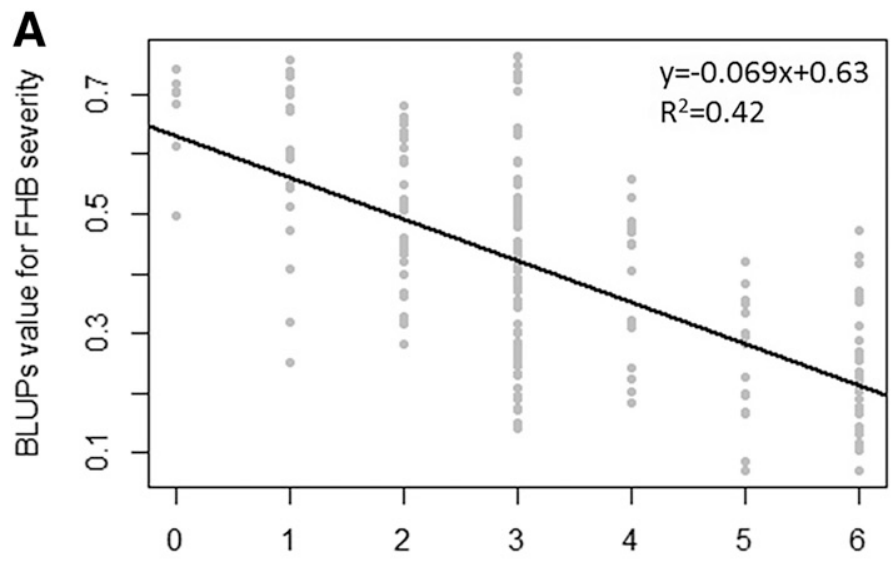

The number of resistant loci

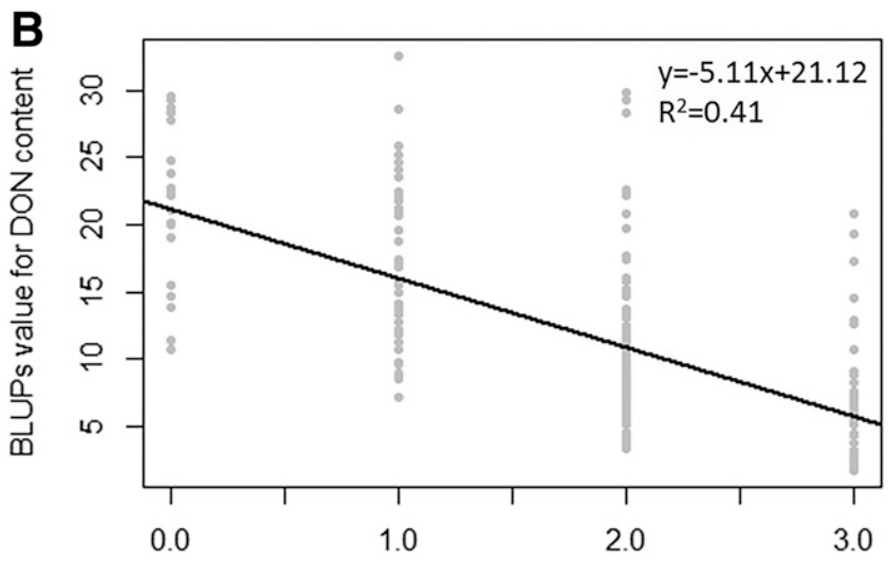

The number of resistant loci

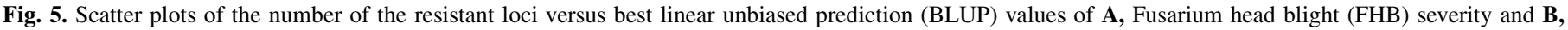
deoxynivalenol (DON) content across the different environments in all 213 wheat accessions. 
resistance, and QFHB-2BL.2 (681.5 to $682 \mathrm{Mb}$ ) was only associated with type II resistance (Table 2). Wang et al. (2017) reported that Qfhb-2B-1 (545.3 to $546.2 \mathrm{Mb}$ ) was significantly associated with type I resistance, and Qfhb-2B-2 (181.6 to 212.6 $\mathrm{Mb}$ ) and Qfhb-2B-3 (758.6 to $763.6 \mathrm{Mb}$ ) were associated with type III resistance in CIMMYT germplasms (Wang et al. 2017). QFHB2BL.2 (681.5 to $682 \mathrm{Mb}$ ) detected in this study might be adjacent to the locus Qfhb-2B-3 (758.6 to $763.6 \mathrm{Mb}$ ), but they had a different response to FHB. A QTL with the flanking markers Xgwm501 and Xgwm47 (672.1 to $685.8 \mathrm{Mb}$ ) was associated with type II resistance in the European winter population (G16-92/Hussar), which explained $14.1 \%$ of the phenotypic variance (Buerstmayr et al. 2009). This QTL partially overlapped with the chromosome region of QFHB-2BL.2.

QFHB-3A (13 Mb), linked by single marker IWB50548, was associated with type II and III resistance. In previous reports, multiple resistant loci $(552.7$ to $702.9 \mathrm{Mb})$ were identified on chromosome 3A with the flanking markers Xwmc264, Xwmc428, Xdupw227, Xgwm720, and Xgwm155. Considering that there was a long distance in the physical map between QFHB-3A and the loci reported in the previous study, QFHB-3A could be a novel locus for type II and III resistance.

The major FHB resistance QTL Fhb1 (7.6 to 13.9Mb) on 3BS was associated with type I, II, and III resistance (Buerstmayr et al. 2009). Rawat et al. (2016) previously reported that a pore-forming toxinlike gene ( $T a P F T)$ at the Fhb1 region confers FHB resistance. In the present study, QFHB-3B.1 (8.1 Mb) was located in the Fhb1 region and its physical location was close to the gene TaPFT. QFHB-3B.2 $(2.0 \mathrm{Mb}$ ) linked with single SNP marker IWB10842 could be a novel resistant locus because no other QTLs or genes have yet been reported in this chromosome region.

QFHB-7A (638.7 to $671.0 \mathrm{Mb}$ ) partially overlapped with the position of previously reported QTLs flanked by Xgwm276 and Xgwm282 (642.9 to 681.4 Mb) for type II resistance (Buerstmayr et al. 2009; Semagn et al. 2007). In our study, QFHB-7A (638.7 to $671.0 \mathrm{Mb}$ ) was detected in a large interval for type III resistance, and further investigation was needed to validate whether QFHB-7A was a pleiotropic locus or a cluster of different resistant genes.

The mean level of FHB resistance was higher in YT than that in the other groups $(P<0.05)$ (Fig. 3C and D). The imbalanced distribution of favorable alleles (Table 3) might explain the different level of FHB resistance in different agroecological wheat growing regions. QFHB-1A, QFHB-2BL.1, QFHBL.2, and QFHB-7A were predominant loci in YT (Table 3) and these resistant loci might be pyramided in traditional breeding programs. Screening of resistant sources and development of FHB resistant cultivars has a long tradition in the lower Yangtze valley region and was initiated in the mid-1970s. Some good FHB resistance sources, such as Sumai3, Ning7840, and Wangshuibai, were developed during that time and were used for FHB resistance improvement worldwide. Series of cultivars Ningmai and Shengkang, which were developed through phenotypic selection, MAS, or somaclonal mutagenesis, showed moderate resistance to FHB spread or DON accumulation.

Pyramiding the favorable alleles could be useful to control FHB epidemics and reduce DON contamination in grains (Arruda et al. 2016; Wang et al. 2017). Eight cultivars (Hong5002, Shengkang2, Ningmai9, Ningmai13, Ningmai15, Ningfeng518, Shengxuan4, and Xiangmai48), which were highly resistant in our field test, all carried the beneficial alleles at QFHB-1A, QFHB-2B.1, QFHB-3A, QFHB-3B.2, QFHB-2BL.1, QFHB3B.1, and QFHB-7A.These accessions had improved agronomic traits and could accelerate the process of resistant allele introgression for controlling FHB epidemics and reducing DON contamination in grains.

This study explored the genetic composition of the elite Chinese wheat germplasm including landraces, breeding lines, and commercial cultivars and identified seven MTAs for FHB resistance that were consistently detected in multiple environments. Four MTAs (QFHB-1A, QFHB-2B.1, QFHB-3A, and QFHB-3B.2) were novel QTLs for FHB resistance, and the other three (QFHB-2BL.1, QFHB3B.1, and QFHB-7A) overlapped with the regions containing previously reported QTLs. There was a cluster of SNPs in QFHB$1 \mathrm{~A}$ and QFHB-2B.1, respectively, which might contribute to MAS for FHB resistance improvement.

\section{LITERATURE CITED}

Adhikari, T. B., Jackson, E. W., Gurung, S., Hansen, J. M., and Bonman, J. M. 2011. Association mapping of quantitative resistance to Phaeosphaeria nodorum in spring wheat landraces from the USDA National Small Grains Collection. Phytopathology 101:1301-1310.

Anderson, J. A., Chao, S., and Liu, S. 2007. Molecular breeding using a major QTL for Fusarium head blight resistance in wheat. Crop Sci. 47(suppl. 3):S-112-S-119.

Arruda, M. P., Brown, P., Brown-Guedira, G., Krill, A. M., Thurber, C., Merrill, K. R., Foresman, B. J., and Kolb, F. L. 2016. Genome-wide association mapping of Fusarium head blight resistance in wheat using genotyping-by-sequencing. Plant Genome 9. https://doi.org/10.3835/ plantgenome2015.04.0028.

Bai, G., and Shaner, G. 2004. Management and resistance in wheat and barley to Fusarium head blight. Annu. Rev. Phytopathol. 42:135-161.

Breseghello, F., and Sorrells, M. E. 2006. Association mapping of kernel size and milling quality in wheat (Triticum aestivum L.) cultivars. Genetics 172: 1165-1177.

Buerstmayr, H., Ban, T., and Anderson, J. A. 2009. QTL mapping and markerassisted selection for Fusarium head blight resistance in wheat: A review. Plant Breed. 128:1-26.

Buerstmayr, H., Lemmens, M., Hartl, L., Doldi, L., Steiner, B., Stierschneider, M., and Ruckenbauer, P. 2002. Molecular mapping of QTLs for Fusarium head blight resistance in spring wheat. I. Resistance to fungal spread (type II resistance). Theor. Appl. Genet. 104:84-91.

Buerstmayr, H., Steiner, B., Hartl, L., Griesser, M., Angerer, N., Lengauer, D., Miedaner, T., Schneider, B., and Lemmens, M. 2003. Molecular mapping of QTLs for Fusarium head blight resistance in spring wheat. II. Resistance to fungal penetration and spread. Theor. Appl. Genet. 107:503-508.

Chen, G., Zhang, H., Deng, Z., Wu, R., Li, D., Wang, M., and Tian, J. 2016. Genome-wide association study for kernel weight-related traits using SNPs in a Chinese winter wheat population. Euphytica 212:173-185.

del Blanco, I., Frohberg, R., Stack, R., Berzonsky, W., and Kianian, S. 2003. Detection of QTL linked to Fusarium head blight resistance in Sumai 3derived North Dakota bread wheat lines. Theor. Appl. Genet. 106:1027-1031.

Dodig, D., Zoric, M., Kobiljski, B., Savic, J., Kandic, V., Quarrie, S., and Barnes, J. 2012. Genetic and association mapping study of wheat agronomic traits under contrasting water regimes. Int. J. Mol. Sci. 13:6167-6188.

Escrivá, L., Font, G., and Manyes, L. 2015. In vivo toxicity studies of Fusarium mycotoxins in the last decade: A review. Food Chem. Toxicol. 78: 185-206.

Fuentes, R. G., Mickelson, H. R., Busch, R. H., Dill-Macky, R., Evans, C. K., Thompson, W. G., Wiersma, J. V., Xie, W., Dong, Y., and Anderson, J. A. 2005. Resource allocation and cultivar stability in breeding for Fusarium head blight resistance in spring wheat. Crop Sci. 45:1965-1972.

Jiang, G. L., Dong, Y., Shi, J., and Ward, R. W. 2007a. QTL analysis of resistance to Fusarium head blight in the novel wheat germplasm CJ 9306. II. Resistance to deoxynivalenol accumulation and grain yield loss. Theor. Appl. Genet. 115:1043-1052.

Jiang, G. L., Shi, J., and Ward, R. W. 2007b. QTL analysis of resistance to Fusarium head blight in the novel wheat germplasm CJ 9306. I. Resistance to fungal spread. Theor. Appl. Genet. 116:3-13.

Kollers, S., Rodemann, B., Ling, J., Korzun, V., Ebmeyer, E., Argillier, O., Hinze, M., Plieske, J., Kulosa, D., Ganal, M. W., and Roder, M. S. 2013. Whole genome association mapping of Fusarium head blight resistance in European winter wheat (Triticum aestivum L.). PLoS One 8:e57500.

Kulwal, P., Ishikawa, G., Benscher, D., Feng, Z., Yu, L. X., Jadhav, A., Mehetre, S., and Sorrells, M. E. 2012. Association mapping for pre-harvest sprouting resistance in white winter wheat. Theor. Appl. Genet. 125: 793-805.

Letta, T., Maccaferri, M., Badebo, A., Ammar, K., Ricci, A., Crossa, J., and Tuberosa, R. 2013. Searching for novel sources of field resistance to Ug99 and Ethiopian stem rust races in durum wheat via association mapping. Theor. Appl. Genet. 126:1237-1256.

Lipka, A. E., Tian, F., Wang, Q., Peiffer, J., Li, M., Bradbury, P. J., Gore, M. A., Buckler, E. S., and Zhang, Z. 2012. GAPIT: Genome association and prediction integrated tool. Bioinformatics 28:2397-2399.

Liu, S., and Anderson, J. A. 2003. Marker assisted evaluation of Fusarium head blight resistant wheat germplasm. Crop Sci. 43:760-766.

Liu, S., Hall, M. D., Griffey, C. A., and McKendry, A. L. 2009. Meta-analysis of QTL associated with Fusarium head blight resistance in wheat. Crop Sci. 49:1955-1968. 
Ma, H. X., Bai, G. H., Zhang, X., and Lu, W. Z. 2006. Main effects, epistasis, and environmental interactions of quantitative trait loci for Fusarium head blight resistance in a recombinant inbred population. Phytopathology 96:534-541.

Ma, H. X., Yao, J. B., Zhou, M. P., Zhang, X., Ren, L. J., Yu G. H., and Lu, W. Z. 2008. Molecular breeding for wheat Fusarium head blight resistance in China. Cereal Res. Commun. 36(suppl. B):203-212.

Maccaferri, M., Zhang, J., Bulli, P., Abate, Z., Chao, S., Cantu, D., Bossolini, E., Chen, X., Pumphrey, M., and Dubcovsky, J. 2015. A genome-wide association study of resistance to stripe rust (Puccinia striformis $\mathrm{f}$. sp. tritici) in a worldwide collection of hexaploid spring wheat (Triticum aestivum L.). G3 Genes Genomes Genet. 5:449-465.

McMullen, M., Bergstrom, G., De Wolf, E., Dill-Macky, R., Hershman, D., Shaner, G., and Van Sanford, D. 2012. A unified effort to fight an enemy of wheat and barley: Fusarium head blight. Plant Dis. 96:1712-1728.

Mesterházy, A. 1995. Types and components of resistance to Fusarium head blight of wheat. Plant Breed. 114:377-386.

Mesterházy, Á., Bartók, T., Mirocha, C. G., and Komoróczy, R. 1999. Nature of wheat resistance to Fusarium head blight and the role of deoxynivalenol for breeding. Plant Breed. 118:97-110.

Miedaner, T., and Korzun, V. 2012. Marker-assisted selection for disease resistance in wheat and barley breeding. Phytopathology 102:560-566.

Miedaner, T., Würschum, T., Maurer, H. P., Korzun, V., Ebmeyer, E., and Reif, J. C. 2011. Association mapping for Fusarium head blight resistance in European soft winter wheat. Mol. Breed. 28:647-655.

Mohan, A., Kulwal, P., Singh, R., Kumar, V., Mir, R. R., Kumar, J., Prasad, M., Balyan, H. S., and Gupta, P. K. 2009. Genome-wide QTL analysis for preharvest sprouting tolerance in bread wheat. Euphytica 168:319-329.

Naruoka, Y., Garland-Campbell, K. A., and Carter, A. H. 2015. Genome-wide association mapping for stripe rust (Puccinia striiformis $\mathrm{f}$. sp. tritici) in US Pacific Northwest winter wheat (Triticum aestivum L.). Theor. Appl. Genet. 128:1083-1101.

R Development Core Team. 2011. R: A Language and Environment for Statistical Computing. R Foundation for Statistical Computing, Vienna, Austria. http://www.R-project.org/

Rawat, N., Pumphrey, M. O., Liu, S., Zhang, X., Tiwari, V. K., Ando, K., Trick, H. N., Bockus, W. W., Akhunov, E., Anderson, J. A., and Gill, B. S. 2016. Wheat Fhb1 encodes a chimeric lectin with agglutinin domains and a pore-forming toxin-like domain conferring resistance to Fusarium head blight. Nat. Genet. 48:1576-1580.

Reif, J. C., Gowda, M., Maurer, H. P., Longin, C. F., Korzun, V., Ebmeyer, E., Bothe, R., Pietsch, C., and Wurschum, T. 2011. Association mapping for quality traits in soft winter wheat. Theor. Appl. Genet. 122:961-970.

Schweiger, W., Steiner, B., Vautrin, S., Nussbaumer, T., Siegwart, G., Zamini, M., Jungreithmeier, F., Gratl, V., Lemmens, M., Mayer, K. F., Berges, H.,
Adam, G., and Buerstmayr, H. 2016. Suppressed recombination and unique candidate genes in the divergent haplotype encoding Fhb1, a major Fusarium head blight resistance locus in wheat. Theor. Appl. Genet. 129: 1607-1623.

Semagn, K., Skinnes, H., Bjørnstad, Å., Marøy, A. G., and Tarkegne, Y. 2007. Quantitative trait loci controlling Fusarium head blight resistance and low deoxynivalenol content in hexaploid wheat population from 'Arina' and NK93604. Crop Sci. 47:294-303.

Wang, R., Chen, J., Anderson, J. A., Zhang, J., Zhao, W., Wheeler, J., Klassen, N., See, D. R., and Dong, Y. 2017. Genome-wide association mapping of Fusarium head blight resistance in spring wheat lines developed in the Pacific Northwest and CIMMYT. Phytopathology 107:1486-1495.

Wang, S., Wong, D., Forrest, K., Allen, A., Chao, S., Huang, B. E., Maccaferri, M., Salvi, S., Milner, S. G., Cattivelli, L., Mastrangelo, A. M., Whan, A., Stephen, S., Barker, G., Wieseke, R., Plieske, J., Lillemo, M., Mather, D., Appels, R., Dolferus, R., Brown-Guedria, G., Korol, A., Akhunova, A. R., Feuillet, C., Salse, J., Morgante, M., Pozniak, C., Luo, M., Dvorak, J., Morell, M., Dubcovsky, J., Ganal, M., Tuberosa, R., Lawley, C., Mikoulitch, I., Cavanagh, C., Edwards, K. J., Hayden, M., and Akhunov, E.; International Wheat Genome Sequencing Consortium. 2014. Characterization of polyploid wheat genomic diversity using a high-density 90000 single nucleotide polymorphism array. Plant Biotechnol. J. 12:787-796.

White, J., Law, J. R., MacKay, I., Chalmers, K. J., Smith, J. S. C., Kilian, A., and Powell, W. 2008. The genetic diversity of UK, US and Australian cultivars of Triticum aestivum measured by DArT markers and considered by genome. Theor. Appl. Genet. 116:439-453.

Wilde, F., Korzun, V., Ebmeyer, E., Geiger, H. H., and Miedaner, T. 2007. Comparison of phenotypic and marker-based selection for Fusarium head blight resistance and DON content in spring wheat. Mol. Breed. 19: 357-370.

Yang, Z., Gilbert, J., Fedak, G., and Somers, D. J. 2005. Genetic characterization of QTL associated with resistance to Fusarium head blight in a doubled-haploid spring wheat population. Genome 48:187-196.

Zegeye, H., Rasheed, A., Makdis, F., Badebo, A., and Ogbonnaya, F. C. 2014. Genome-wide association mapping for seedling and adult plant resistance to stripe rust in synthetic hexaploid wheat. PLoS One 9:e105593.

Zhang, D., Bai, G., Zhu, C., Yu, J., and Carver, B. F. 2010a. Genetic diversity, population structure, and linkage disequilibrium in U.S. elite winter wheat. Plant Genome 3:117-127.

Zhang, Z., Ersoz, E., Lai, C. Q., Todhunter, R. J., Tiwari, H. K., Gore, M. A., Bradbury, P. J., Yu, J., Arnett, D. K., Ordovas, J. M., and Buckler, E. S. 2010b. Mixed linear model approach adapted for genome-wide association studies. Nat. Genet. 42:355-360. 